Pacific Journal of Mathematics

ON THE RELATIONSHIP OF A RING AND THE SEBRING 


\title{
ON THE RELATIONSHIP OF A RING AND THE SUBRING GENERATED BY ITS SYMMETRIC ELEMENTS
}

\section{Charles LANSKI}

\begin{abstract}
Let $R$ be a ring with involution, and $\bar{S}$ the subring generated by the symmetric elements of $R$. By placing various conditions on the elements of $\bar{S}$, it is shown that the same conditions are forced on $R$. For example, if $\bar{S}$ is nil or algebraic, then so is $R$. Also, if $R$ is assumed to be simple, prime, or semi-prime, then $\bar{S}$ satisfies the same property. Lastly, each of these three conditions on $\bar{S}$ implies the same property for $R$, modulo a nilpotent ideal of $R$.
\end{abstract}

I. $R$ will always denote an associative ring with involution, * Thus, * is an anti-automorphism of $R$ of period 2. Let $S(R)=$ $\left\{r \in R \mid r^{*}=r\right\}$ denote the set of symmetric elements of $R$. When there can be no confusion, we write $S$ in place of $S(R)$. The symbol $\bar{S}$ will represent the subring generated by $S$, and $Z$ will represent the center of $R$. For $x, y \in R$, let $[x, y]=x y-y x$. An additive subgroup $U$ of $R$ is called a Lie ideal of $R$ if $[u, r] \in U$ for all $u \in U$ and $r \in$ $R$.

It will be necessary to refer frequently to some well-known results, most of which can be found in [3]. For the sake of completeness and convenience, we present these results in a form more useful for our purposes. The first two tell us when $\bar{S}$ contains an ideal of $R$.

Lemma 1.1. $\bar{S}$ is a Lie ideal of $R$.

Proof. We use the proof of Lemma 1 of [5]. Suppose $s \in S$ and $r \in R$. Then $s r-r s=s r+r^{*} s-\left(r+r^{*}\right) s \in \bar{S}$. If we assume that for $s_{1}, \cdots, s_{k-1} \in S$ and $r \in R$ that $\left[s_{1} s_{2} \cdots s_{k-1}, r\right] \in \bar{S}$, then by induction we have $\left[s_{1} s_{2} \cdots s_{k}, r\right]=s_{1} s_{2} \cdots s_{k-1}\left[s_{k}, r\right]+\left[s_{1} s_{2} \cdots s_{k-1}, r\right] s_{k} \in \bar{S}$ for all $k$. Since every element of $\bar{S}$ is a sum of such terms, the lemma is proved.

LEMMA 1.2. If $U$ is a noncommutative subring and Lie ideal of $R$, then $U$ contains a nonzero ideal of $R$.

Proof. This is essentially Lemma 1.3 of [3]. Let $a, b \in U$ so that $[a, b] \neq 0$. Let $r \in R$. Then $[a, r b]=r[a, b]+[a, r] b$. Since $[a, r] \in$ $U$ and $U$ is a subring, we have $r[a, b] \in U$. For $t \in R[r[a, b], t] \in U$. Thus $r[a, b] t-\operatorname{tr}[a, b] \in U$, and so $r[a, b] t \in U$. Consequently, $U$ contains the ideal generated by $[a, b] \neq 0$. 
The next lemma, together with Lemma 1.1, gives us information when $\bar{S}$ is commutative. The lemma is essentially the first half of Lemma 1.3 of [3].

LEMmA 1.3. Suppose $U$ is a Lie ideal of $R$ with $[U, U]=0$. If $2 R=0$ and $x \in U$ then $x^{2} \in Z$. If $R$ is semi-prime and 2-torsion-free, then $U \subset Z$.

Proof. If $2 R=0$ then $x \in U$ says $[x,[x, r]]=x^{2} r+r x^{2}=0$ for all $r \in R$. Thus $x^{2} \in Z$.

Suppose that $R$ is semi-prime and 2-torsion free. For $u \in U$ and $x, y \in R$, we have $[u, x y] \in U$, so $0=[u,[u, x y]]=[u, x[u, y]]+[u,[u, x] y]$. Thus $2[u, x][u, y]=0$, and so $[u, x][u, y]=0$. Letting $y=r x$ for $r \in R$ gives $[u, x] R[u, x]=0$. Since $R$ is semi-prime, $[u, x]=0$, so $u \in Z$.

In the study of rings with involution, often one can prove certain facts if either $2 R=0$, or if $R$ is 2 -torsion free. This situation arises in considering those properties in which we are interested, namely, the properties of being nil, locally nilpotent, algebraic, and locally algebraic. To enable us to reduce the general case to one of the two special situations of $2 R=0$ or $R$ 2-torsion free, we introduce the following formalization:

Given a ring $A$, let $F(A)$ denote the set of finite subsets of $A$. Let $P$ be a property of the elements of $F(A)$, satisfying:

(1) If $\left\{A_{i}\right\}$ is a collection of rings and the elements of $F\left(A_{i}\right)$ satisfy $P$ for each $i$, then the elements of $F\left(\oplus A_{i}\right)$ satisfy $P$, where $\bigoplus A_{i}$ is the direct sum of the $A_{i}$.

(2) If $B$ is an ideal of $A$ and the elements of both $F(B)$ and $F(A / B)$ satisfy $P$, then so do the elements of $F(A)$.

Note that the four properties mentioned above are properties of finite subsets, and satisfy the two conditions stated.

Now we can state the lemma which will enable us to reduce the general case to the special cases cited above.

LEMMA 1.4. For all rings $B$ with either $2 B=0$, or $B$ 2-torsion free, let $P$ be a property of the elements of $F(B)$ satisfying the two conditions above. Then $P$ is a property of the elements of $F(A)$ for any $\operatorname{ring} A$.

Proof. Let $A$ be a ring, $T(A)$ the torsion ideal of $A$, and $T_{p}(A)$ the $p$-primary component of $T(A)$. Since $A / T_{2}(A)$ is 2-torsion free, $P$ is a property of the elements of $F\left(A / T_{2}(A)\right)$. Thus, it suffices to show 
that $P$ is a property of the elements of $F\left(T_{2}(A)\right)$. Let $B=T_{2}(A)$, and $B_{k}=\left\{x \in B \mid 2^{k} x=0\right\}$. Now $2 B_{1}=0$ and $2\left(B_{2} / B_{1}\right)=0$, so $P$ is a property of the elements of $F\left(B_{2}\right)$. By induction, $P$ is a property of the elements of $F\left(B_{k}\right)$ for any $k$. Hence, $P$ is a property of the elements of $F(B)$, for $F(B)$ is the union of the $F\left(B_{k}\right)$ for all $k$.

II. In this section we consider the situation when $\bar{S}$ is nil, locally nilpotent, algebraic, locally finite, and nilpotent. Clearly, if $R$ satisfies any of these conditions, then so does $\bar{S}$. The converse holds, except for $\bar{S}$ nilpotent and $2 R=0$.

One technique that we shall use often in this section involves considering certain homomorphic images of $R$. In general, a homomorphic image of a ring with involution need not have an involution itself. However, if $A$ is an ideal of $R$ and $A^{*}=A$, then the quotient $R / A$ inherits an involution from $R$ by defining $(r+A)^{*}=$ $r^{*}+A$. It is this involution on $R / A$ to which we shall refer in this section.

We begin by eliminating the trivial case.

Lemma 2.1. If $S=0$ then $R^{3}=0$.

Proof. Let $I=\{r \in R \mid 2 r=0\} . \quad I$ is an ideal of $R$ and $I^{*}=I$. If $x \in I$ then $x+x^{*} \in S$, so $x+x^{*}=0$. But $2 x=0$, so $x=x^{*}$. Thus $I=0$ and $R$ is 2-torsion free. For $x \in R x+x^{*}, x x^{*} \in S$ so $0=x x^{*}=$ $-x^{2}$. But if $R$ is 2-torsion free and nil of index 2, we must have $R^{3}=0$.

Our first main result is a complete answer when $\bar{S}$ is nil.

THEOREM 2.2. If $\bar{S}$ is nil then $R$ is nil.

Proof. By Lemma 1.4, it suffices to assume that either $R$ is 2torsion free or $2 R=0$. In either case, let $N$ be the sum of all nil ideals of $R$. Clearly $N^{*}=N$, so $R / N$ has an induced involution, and further, contains no nonzero nil ideals. Also, if $R$ is 2-torsion free then so is $R / N$, for $T=\{x \in R \mid 2 x \in N\}$ is a nil ideal of $R$, so is contained in $N$. If $\rho: R \rightarrow R / N$ is the natural homomorphism, then $\rho(\bar{S})$ is a subring and Lie ideal of $R / N$. Suppose that $\rho(\bar{S})$ is not commutative. By Lemma $1.2 \rho(\bar{S})$ would contain a nonzero ideal of $R / N$, which is impossible since $\rho(\bar{S})$ is nil. Thus $\rho(\bar{S})$ is commutative. Whether $R$ is 2 -torsion free or $2 R=0$, we can conclude, using Lemma 1.3, that $\rho(x)^{2} \in Z(R / N)$ for $x \in \bar{S}$. But a nil element in the center of any ring generates a nilpotent ideal. Hence $\rho(x)^{2}=0$. Thus $[\rho(x), \rho(r)]^{2} \rho(x)=0$ for all $r \in R$ and any $x \in \bar{S}$. This implies that 
$\rho(x R)$ is nil of index 3. Since $R / N$ is semi-prime we must have $\rho(x)=$ 0 , using Levitzki's theorem [3, Lemma 1.1]. But then if $r \in R$ we have $0=\rho\left(r r^{*}\right)=\rho(r) \rho\left(r^{*}\right)$ and $0=\rho\left(r+r^{*}\right)=\rho(r)+\rho\left(r^{*}\right)$. Thus $R / N$ is nil of index 2 , so $R$ must be nil.

Using the same technique but assuming a bit more gives

THEOREM 2.3. If $\bar{S}$ is locally nilpotent, so is $R$.

Proof. Proceed exactly as in Theorem 2.2 using $\mathscr{L}(R)$, the sum of all locally nilpotent ideals [4, p. 197], in place of $N$.

As we have mentioned, $\bar{S}$ nilpotent does not in general imply that $R$ is nilpotent. However, when $R$ is 2 -torsion free it is nilpotent, and we can obtain a bound on the index of nilpotence.

THEOREM 2.4. If $R$ is 2-torsion free and $\bar{S}^{n}=0$, then $R^{3 n}=0$.

Proof. By Lemma $1.1 \bar{S}$ is a Lie ideal of $R$. Let $t_{m}=s_{1} s_{2} \cdots s_{m}$ for $s_{i} \in S$. Then for $r \in R$

$$
\left[t_{m}, r\right]=\sum_{i=1}^{m} s_{1} \cdots s_{i-1}\left[s_{i}, r\right] s_{i+1} \cdots s_{m} \in S^{m}+S^{m+1}
$$

since $\left[s_{i}, r\right]=s_{i} r+r^{*} s_{i}-\left(r+r^{*}\right) s_{i} \in S+S^{2}$. Thus $\left[t_{m}, r\right] t_{n-m}=t_{m} r t_{n-m}=0$. Repeating the argument on $t_{m}$ and $t_{n-m}$ gives $s_{1} r_{1} s_{2} \cdots r_{n-1} s_{n}=0$ for $s_{j} \in S$ and $r_{i} \in R$ or $r_{i}$ formally equal to 1 . Therefore, the ideal $I$ generated by $S$ in nilpotent of index $n$. If $r \in R$ then $r r^{*}, r+r^{*} \epsilon$ $S \subset I$. This gives $2 R^{3} \subset I$ or $2^{n} R^{3 n}=0$. Since $R$ is 2 -torsion free, $R^{3 n}=0$.

The proof of Theorem 2.4 fails in the case where $2 R=0$ at the point where we obtain $R / I$ is a nil ring of index 2. Of course, one can have such a non-nilpotent ring in the characteristic 2 case. An example would be a polynomial ring in infinitely many indeterminates over $G F(2)$, modulo all squares. We present such an example with involution which will show that Theorem 2.4 is false if $2 R=0$. Thanks are due to Dennis Estes for conversations regarding the existence of an example and particularly for his suggestion to view the involution as we will, which makes verification of the example relatively simple.

Let $X=\left\{x_{i}\right\}$ and $Y=\left\{y_{i}\right\}$ be countable sets of indeterminates indexed by positive integers, and set $T$ equal to the ring of polynomials in $X$ and $Y$ with coefficients in $G F(2)$ whose constant terms are zero. Let $I$ be the ideal of $T$ generated by all $x_{i}^{2}$ and $y_{i} y_{j}$, and let $R=T / I$. Denote the image of $x_{i}$ by $\bar{x}_{i}$ and of $y_{i}$ by $\bar{y}_{i}$. Lastly, 
denote by $D_{i}$ the formal partial derivative on $R$ with respect to $\bar{x}_{i}$. $R$ has an involution defined by $\bar{x}_{i}^{*}=\bar{x}_{i}+\bar{y}_{i}$ and $\bar{y}_{i}^{*}=\bar{y}_{i}$. If $f(\bar{Y})$ is a polynomial in the $\left\{\bar{y}_{i}\right\}$ alone, then clearly $f(\bar{Y})^{*}=f(\bar{Y})$. If $f(\bar{X})$ is a polynomial in the $\left\{\bar{x}_{i}\right\}$ then since $\bar{y}_{i} \bar{y}_{j}=0$, we have $f(\bar{X})^{*}=f(\bar{X})+$ $\sum \bar{y}_{i} D_{i} f(\bar{X})$. Thus $\left(f(\bar{X}) \bar{y}_{i}\right)^{*}=\left(f(\bar{X})+\sum \bar{y}_{i} D_{i} f(\bar{X})\right) \bar{y}_{i}=f(\bar{X}) \bar{y}_{i}$, and so $S$ contains the ideal generated by all $\bar{y}_{i}$. Suppose that $f(\bar{X}) \in S$. Then by above we must have $\sum \bar{y}_{i} D_{i} f(\bar{X})=0$, and so, if we take a polynomial $f(X) \in T$ which maps to our element $f(\bar{X}) \in R$ we have $\sum y_{i} D_{i} f(X)=\sum p_{i}(X, Y) x_{i}^{2}+\sum q_{i j}(X, Y) y_{i} y_{j}$ where we may assume that $D_{i} f(X)$ has no monomials containing any $x_{j}^{2}$, and where $p_{i}$ and $q_{i j}$ may have a nonzero constant term. By looking at degrees of elements of $T$ in each $y_{i}$, and then in the other $y_{j}$, we obtain $y_{i} D_{i} f(X)=\sum p_{i}(X, Y) x_{i}^{2}$. Now consider the degrees of both sides with respect to the $x_{j}$. The result is that $y_{i} D_{i} f(X)=0$. Since $T$ is a domain we must have $D_{i} f(X)=0$. But then no $x_{i}$ appears in any monomial of $f(X)$; that is, $f(X)=0$. This shows that in $R, S=\left(\bar{y}_{i}\right)$, the ideal generated by the $\left\{\bar{y}_{i}\right\}$. Clearly $S^{2}=0$ but $R$ is not nilpotent, since $R / S \cong G F(2)^{\prime}[X] \mid\left(x_{i}^{2}\right)$, where $G F(2)^{\prime}[X]$ are the polynomials in $X$ without constant terms.

To obtain a noncommutative counterexample to Theorem 2.4 take $R_{n}$, the complete $n \times n$ matrix ring over $R$, the commutative example above, and consider the subring $W=\left\{\left(a_{i j}\right) \in R_{n} \mid a_{i j} \in S\right.$ for $\left.i \neq j\right\}$. Define $\left(a_{i j}\right)^{*}=\left(a_{j i}^{*}\right)$. Thus, the involution on $W$ takes transposes and applies * from $R$. Again, $W$ is not nilpotent but since $S(W) \subset(S(R))_{n}$ we have $S(W)^{2}=0$.

Assume for the remainder of this section that $R$ is an algebra over a field $F$, and that ${ }^{*}$ is compatible with the action of $F$ in that $(a r)^{*}=a r^{*}$ for $a \in F$.

\section{THEOREM 2.5. If $\bar{S}$ is algebraic over $F$, so is $R$.}

Proof. Let $A$ be the algebraic kernel of $R$; that is, the sum of all algebraic ideals of $R$. Clearly $A^{*}=A$, so $R / A$ inherits the involution from $R$ and contains no algebraic ideals. If $\rho: R \rightarrow R / A$ is the natural homomorphism, we have, as in Theorem 2.2, that $\rho(\bar{S})$ is a Lie ideal and subring of $R / A$. If $\rho(\bar{S})$ is not commutative it must contain a nonzero ideal of $R / A$ by Lemma 1.2. But this ideal would be algebraic over $F$, which is impossible. Hence $\rho(\bar{S})$ is commutative. Given any $x \in R$ we have $\rho(x)^{2}-\rho\left(x+x^{*}\right) \rho(x)+\rho\left(x^{*} x\right)=0$, where $\rho\left(x+x^{*}\right)$ and $\rho\left(x^{*} x\right)$ are algebraic over $F$. Thus to show that $R / A$, and so $R$ is algebraic over $F$, it is enough to do so for an algebra $B$ with the property that for each $x \in B, x^{2}+b x+c=0$ for some $b, c \in$ $B$ with $b$ and $c$ commuting with each other and each algebraic over $F$. Let $F[b, c]=C$ and $[C: F]=n$. Since $x^{2}=-b x-c$ we have 
$x^{3}=-b x^{2}-c x=-b(-b x-c)-c x$, and so $x^{3}=b_{2} x+c_{2}$ for $b_{2}, c_{2} \in C$. Similarly one obtains $x^{k}=b_{k-1} x+c_{k-1}$ for all $k \geqq 2$. Now the collection $\left\{b_{i}\right\}$ is linearly dependent over $F$. Let $\sum_{i=1}^{m} f_{i} b_{i}=0$ be a nontrivial dependence relation, and set $g(Y)=\sum_{i=1}^{m} f_{i} Y^{i+1}$. Then $g(x)=$ $\sum f_{i} c_{i}=d \in C$. Let $h(Y)$ be a nontrivial polynomial satisfied by $d$. Then $0=h(d)=h(g(x))$, and so $x$ is algebraic over $F$.

In the same way that we went from nil to locally nilpotent, we may now consider the property of local finiteness, although here we need the result in Theorem 2.5 as well as the method of proof.

\section{THEOREM 2.6. If $\bar{S}$ is locally finite, then so is $R$.}

Proof. The argument is the same as in Theorem 2.5 using $L$, the sum of all the locally finite ideals in place of the sum of all algebraic ideals [2]. Again we may assume that $\rho(\bar{S})$ is commutative and obtain that $R / L$ is algebraic. Since $\rho(\bar{S})$ is commutative, the set of elements of the form $\rho(x)+\rho(x)^{*}$ satisfy a polynomial identity over $F[2]$. By a theorem of Amitsur [1], $R / L$ itself satisfies a polynomial identity. Since $R / L$ is algebraic, it must be locally finite by Theorem 6.4.3 of [2]. Hence $R=L$, so is locally finite.

We mention two examples relevant to the theorems proved in this section. First, if $B$ is any ring and $B^{0 p}$ its opposite ring, then $B \oplus B^{o p}$ has an involution defined by $\left(b_{1}, b_{2}\right)^{*}=\left(b_{2}, b_{1}\right)$. Clearly, $S\left(B \oplus B^{0 p}\right)=\{(b, b) \mid b \in B\}$. This example shows that if $\bar{S}$ satisfies one of the properties we have considered, one cannot hope to force $R$ to satisfy a stronger property, in the sense, for example, that nilpotence is a stronger property than local nilpotence.

The second example concerns the following situation. If $R$ is 2torsion free and $k=\left\{r \in R \mid r^{*}=-r\right\}$ then $2 R \subseteq S+K$ and $S \cap K=0$. One might ask whether imposing conditions on $\bar{K}$ as we did on $\bar{S}$ will also force these conditions on $R$. In this case the answer is no. Let $A$ be a commutative ring and $N$ an ideal of $A$. If $A_{n}$ is the complete $n \times n$ matrix ring over $A$, consider $R=\left\{\left(a_{i j}\right) \in A_{n}\right\} a_{i j} \in N$ for $i \neq j\}$. Let $\left(a_{i j}\right)^{*}=\left(a_{j i}\right)$. If $A$ is 2-torsion free then $\bar{K} \subset N_{n}$. In particular, $N$ may be nil, nilpotent, or algebraic, and so $\bar{K}$ has the same property, but $R$ may fail to. Specifically, let $A=F[x, y] /\left(x^{t}\right)$ and $N=(x)$. Then $\bar{K}$ is nilpotent but $R$ is not even nil, or algebraic. One would like to have such examples which do not satisfy some polynomial identity. This can be accomplished by considering matrix rings described above and then taking direct sums of such rings with the size of the matrices becoming arbitrarily large.

III. In this section we consider the situation when either $R$ or 
$\bar{S}$ is simple, prime, or semi-prime. The first two lemmas give us information on the "semi-primeness" of $S$.

LEMMA 3.1. If $R$ is semi-prime and $x \in S$ with $x S x=0$, then $x=0$.

Proof. For $r \in R$ we have $x\left(r+r^{*}\right) x=0$, so $x r x=-x r^{*} x$. Hence $x r x r x=-x r x r^{*} x=-x\left(r x r^{*}\right) x=0$, since $r x r^{*} \in S$. Thus, $x R$ is a nil right ideal of $R$ of index 3. By Levitzki's theorem [3, Lemma 1.1] we must have $x=0$.

LEMMA 3.2. If $R$ is semi-prime and $x S x=0$, then $x+x^{*}=0$.

Proof. For $r \in R$ and $s \in S, r x^{*} s+s x r^{*} \in S$. Hence

$$
0=x\left(r x^{*} s+s x r^{*}\right) x=x r x^{*} s x .
$$

Since $R$ is semi-prime, $x^{*} S x=0$. Similarly, $0=x\left(s x^{*} r+r^{*} x s\right) x$ implies $x S x^{*}=0$. As $x^{*} S x^{*}=0$, we have, $\left(x+x^{*}\right) S\left(x+x^{*}\right)=0$. By Lemma 3.1, $x+x^{*}=0$.

The next theorem, that $R$ simple implies that $\bar{S}$ is simple, is an easy consequence of any one of several theorems in [3], together with an examination of the case when $R$ is 4-dimensional over its center and char $R=2$. We prefer to give a proof which depends only on the results presented thus far.

THEOREM 3.3. If $R$ is simple, then either $\bar{S}=R$ or $\bar{S}$ is a field.

Proof. If $\bar{S}$ is not commutative, then by Lemma 1.1 and Lemma 1.2, $\bar{S}$ must contain a nonzero ideal of $R$. Hence $\bar{S}=R$. If $\bar{S}$ is commutative then $\bar{S}=S$ and for $x \in S, x^{2} \in Z$ by Lemma 1.3. If $x \in S$ but $x^{2}=0$, then $x S x=0$ so $x=0$ by Lemma 3.1. Now $S \neq 0$ by Lemma 2.1, so $Z$ is a field. Since every element of $S$ is invertible, $S$ is a field.

The situation when $R$ is prime follows just as easily.

THEOREM 3.4. If $R$ is prime so is $\bar{S}$.

Proof. If $\bar{S}$ is commutative, proceed as in Theorem 3.3 to obtain $x^{2} \in Z-0$ for all $x \in S-0$. Since $R$ is prime, the nonzero elements of $Z$ are not zero divisors in $R$. Thus $S$ is a domain, so is prime. If $\bar{S}$ is not commutative then $\bar{S}$ contains $I \neq 0$ an ideal of $R$. If $A$ and $B$ are ideals of $\bar{S}$ and $A B=0$, then $A I B=0$. Since $R$ is prime, 
either $A=0$ or $B=0$, proving that $\bar{S}$ is prime.

The semi-prime case is a bit more complicated. We need first the following definition.

Definition. If $I$ is an ideal of the semi-prime ring $T$, then Ann $I=\{x \in T \mid I x=0\}$. An ideal $J$ which has the form $J=$ Ann $I$ is called an annihilator ideal of $T$. Note that since $R$ is semi-prime we have $I(\operatorname{Ann} I)=(\operatorname{Ann} I) I=I \cap \operatorname{Ann} I=0$.

\section{THEOREM 3.5. If $R$ is semi-prime then so is $\bar{S}$.}

Proof. If $\bar{S}$ is commutative then it can have no nonzero nilpotent elements by Lemma 3.1, so is semi-prime. If $\bar{S}$ is not commutative then by Lemma $1.2 \bar{S}$ contains the ideal generated by $[a, b]$ for $a, b \in$ $\bar{S}$. Let $I$ be the sum of all such ideals, and note that $I=I^{*}$. If $a, b \in S$ then $a b+I=b a+I$ in $R / I$, so if $x \in \bar{S}$ then $x+I$ is symmetric in $R / I$. Thus $x-x^{*} \in I$, for any $x \in \bar{S}$.

Let $N$ be an ideal of $\bar{S}$ with $N^{2}=0$. Now $N S N \subset N^{2}=0$, so $x+x^{*}=0$ for all $x \in N$ by Lemma 3.2. Further, since $N \bar{S} N=0$ we have $(N I)^{2}=0$. Because $R$ is semi-prime, $N I=0$. If $x \in N$, then we know that $x+x^{*}=0$ and $x-x^{*} \in I$, so $2 x \in I \cap N \subset$ $I \cap$ Ann $I=0$. But $2 x=0$ and $x+x^{*}=0$ imply $x=x^{*} \in S \cap N$. Since $N S N=0$, Lemma 3.1 implies that $N=0$, and so, $\bar{S}$ must be semi-prime.

Although the converses to the last theorems are false, we can obtain reasonable partial converses modulo the existence of a unique maximal nilpotent ideal guaranteed by the next Lemma.

LEMMA 3.6. If $\bar{S}$ is semi-prime then $R$ contains an ideal $N$ with

(1) $N$ is 2-torsion free

(2) $N$ is nil of index 2

(3) $\quad N^{3}=0$

(4) $x \in N$ implies $x+x^{*}=0$

(5) $N$ contains all nilpotent ideals of $R$.

Proof. Suppose that $m$ is a nilpotent ideal of $R$. Since $\bar{S}$ is semi-prime $m \cap S=0$. Also, since $m+m^{*}$ is nilpotent we have $\left(m+m^{*}\right) \cap S=0$. For $x \in m, x+x^{*} \in\left(m+m^{*}\right) \cap S=0$ and $x x^{*} \in$ $m \cap S=0$. Now if $2 x=0$ then since $x+x^{*}=0, x=x^{*} \in S \cap m=0$. Hence $m$ is 2-torsion free, and since $x x^{*}=x+x^{*}=0$, we have $x^{2}=0$ for all $x \in m$. Thus $m^{3}=0$. Let $N$ be the sum of all the nilpotent ideals of $R . \quad N$ is easily seen to satisfy the conditions of the Lemma. 
Let us note that if $2 R=0$ and $\bar{S}$ is semi-prime, then the Lemma implies that $R$ is semi-prime. Also, the same proof can be used to show more. For example, if we assume that $S$ intersects nil ideals, or locally nilpotent ideals, only in zero, then $N$ still exists as indicated and contains all ideals of the type being considered, so they would be nilpotent as well.

Using Lemma 3.6 we can reduce to the situation in which $R$ is semi-prime. To do this consider $R / N$ which is semi-prime and has an induced involution, since $N^{*}=N$. Now by Theorem 3.5 we know that $\overline{S(R / N)}$ is still semi-prime. Unfortunately, even when we can assume that $R$ is semi-prime, $\bar{S}$ prime does not force $R$ to be prime. However, we do have the following theorem.

THEOREM 3.7. If $\bar{S}$ is prime and $R$ is semi-prime, then either $R$ is prime, or $R$ is a subring of $A \oplus A^{0 p}$, where $A=R / P$ is a prime ring, $A^{0 p}$ is the opposite ring of $A, P \cup P^{*}$ contains every annihilator ideal of $R$, and ${ }^{*}$ in $R$ is induced by interchanging co-ordinates in $A \oplus A^{0 p}$.

Proof. Suppose that for every ideal $I \neq 0$ in $R$ we have $I \cap \bar{S} \neq$ 0 . If $B C=0$ for $B$ and $C$ ideals of $R$, then $(B \cap \bar{S})(C \cap \bar{S})=0$, forcing either $B \cap \bar{S}=0$ or $C \cap \bar{S}=0$. Thus either $B=0$ or $C=0$, so $R$ is prime.

Assume now that $R$ is not prime. Let $P$ be an ideal of $R$ maximal with respect to the property that $P \cap \bar{S}=0$. Since $\bar{S}$ is prime, we have by the usual arguments that $P$ is a prime ideal, and so, $P \neq 0$. If $x \in P \cap P^{*}$ then $x x^{*}$ and $x+x^{*}$ are in $P \cap S=0$. Now if $2 x=0$, then $x=x^{*} \in P \cap S=0$. Hence $P \cap P^{*}$ is 2-torsion free. But then $x^{2}=0$ for every $x \in P \cap P^{*}$, so $\left(P \cap P^{*}\right)^{3}=0$. Since $R$ is semi-prime, $P \cap P^{*}=0$. Thus $R$ is a subdirect sum of $R / P$ and $R / P^{*}$. Further $R / P^{*}$ is naturally isomorphic to $(R / P)^{o p}$ via $r+P^{*} \mapsto r^{*}+P$. We can embed $R$ into $R / P \oplus(R / P)^{o p}$ by the map $r \mapsto\left(r+P, r^{*}+P\right)$. Clearly, interchanging co-ordinates in $R / P \oplus(R / P)^{0 p}$ is an involution which restricts to ${ }^{*}$ on $R$. Lastly, suppose $B C=0$ for ideals $B$ and $C$ of $R$. If $B \not \subset P \cup P^{*}$ then since $P$ and $P^{*}$ are prime ideals, $C \subset P \cap P^{*}=$ 0 . Thus every annihilator ideal of $R$ is contained in $P \cup P^{*}$.

As an illustration of Theorem 3.7, consider the ring $A=F[x, y] /(x y)$ where $F[x, y]$ is the full polynomial ring in $x$ and $y$ over an arbitrary field $F$. If $A\left\{w_{1}, w_{2}, w_{3}\right\}$ is the free algebra with 1 over $A$ and $I$ is the ideal in this ring generated by $w_{1}^{2}, w_{2}^{2}$, and $w_{3}^{2}$, let $B=A\left\{w_{1}, w_{2}, w_{3}\right\} / I$. We extend the involution from $A$ to $B$ in the following way. If $a t_{1} t_{2} \cdots t_{n}$ is a monomial in $B$ with $a \in A$ and each $t_{i}$ some $w_{j}$, let $\left(a t_{1} t_{2} \cdots t_{n}\right)^{*}=a^{*} t_{n} t_{n-1} \cdots t_{1}$. One can show that $B$ is a semi-prime 
ring, basically because $b w_{i} b \neq 0$ for each $i$ and $b \neq 0$, but $B$ is not prime since $(x)(y)=0$. Further, since each $w_{i}$ is in $S$ and $x$ and $y$ must both appear in an element of $S$ if either one does, it can be shown that $\bar{S}$ is prime but not a domain. Let $P=(x)$. Then $B$ is a subdirect sum of $B / P$ and $(B / P)^{o p}$, but not a direct sum since $(x)+(y) \neq$ $B$. Also $B / P$ is neither commutative nor a domain.

For an example which is not semi-prime, but where $\bar{S}$ is still prime, let char $F \neq 2$, and let $H$ be the free semi-group with unit generated by $\left\{z_{i}\right\}$ containing more than one indeterminate. Consider the semigroup ring $B[H]$, which can be viewed as the ring of noncommutative polynomials in the $\left\{z_{i}\right\}$ with coefficients in $B$. If $J$ is the ideal of $B[H]$ generated by $\left\{z_{i}^{2}, z_{i} z_{j}+z_{j} z_{i}, w_{k} z_{i}, x z_{i}, y z_{i}\right\}$, let $C=$ $B[H] / J$. Our involution on $B$ can be extended to $C$ by defining $\left(f z_{i}\right)^{*}=-f z_{i}$ for $f \in F$. Now since each $z_{i}$ annihilates "almost everything" in $C$, fails to be symmetric, and since $\left(f z_{i} z_{j}\right)^{*}=f z_{j} z_{i}=-f z_{i} z_{j}$, we have $\overline{S(C)} \cong \overline{S(B)}$. Thus $\overline{S(C)}$ is prime, but the ideal generated by $\left\{z_{i}\right\}$ is the nonzero ideal $N$ of Lemma 3.6 .

Lastly, we examine the situation when $\bar{S}$ is simple. It will be convenient to consider separately the cases where $\bar{S}$ is commutative or not. Since the second case is more straightforward, we consider it first.

THEOREM 3.8. If $\bar{S}$ is simple and not commutative, and $R$ is semi-prime, then $R=\bar{S}$ or $R$ is prime with $\bar{S}$ is unique minimal ideal, $r^{2} \in \bar{S}$ for all $r \in R$ and $Z(R)=0$.

Proof. By Lemma 1.2, $\bar{S}$ contains a nonzero ideal of $R$. Since $\bar{S}$ is simple, it is an ideal of $R$. Consequently, $r^{2}=\left(r+r^{*}\right) r-r^{*} r \in \bar{S}$ for all $r \in R$. Suppose $I \cap \bar{S}=0$ for an ideal $I$ of $R$. Since $x^{2} \in \bar{S}$ for $x \in I, I$ is nil of index 2, contradicting $R$ semi-prime, unless $I=0$. Hence $\bar{S}$ is the intersection of all nonzero ideals of $R$, so is the unique minimal ideal. It follows that $R$ is prime. Lastly, let $x \in Z(R)$. Then $x^{2} \in Z(\bar{S})$, so $\bar{S}$ has an identity if $x \neq 0$. But the identity of $\bar{S}$ is a central idempotent in the prime ring $R$. Thus $1 \in R$, so $\bar{S}=R$ unless $Z(R)=0$.

To see that the second possibility of the theorem can occur, let $B=Q\{x, y\} /(x y-y x-1)$, where $Q\{x, y\}$ is the free algebra with identity over the rational numbers. $B$ is well known to be a simple domain. Let $R=Q x+x B x$. Since $B$ is simple, $x B x$ is simple and an ideal in $R$. Consequently, since $R$ is a domain, $x B x$ is the unique minimal nonzero ideal of $R$. Define ${ }^{*}$ by setting $x^{*}=-x$ and $y^{*}=y$. Clearly $\bar{S}$ contains $x B x$, since it contains some ideal. If $a x+t \in \bar{S}$ for $t \in x B x$, then $a x \in \bar{S}$. But $f(x, y) \in \bar{S}$ implies $\operatorname{deg}_{x} f \neq 1$, so $a=0$ and $\bar{S}=$ $x B x$. 
If $\bar{S}$ is commutative, then $\bar{S}=S$ is a field. In this case, as the next lemma will show, it is always possible to reduce to the situation where $R$ has an identity, a well-defined prime characteristic, and the nonzero elements of $S$ are units in $R$.

LEMmA 3.9. If $S$ is a field then $R=e R \oplus(1-e) R$ where $e$ is the identity of $S$ and a central idempotent in $R$. Further, $(1-e) R$ is a 2-torsion free ideal, nil of index 2.

Proof. Let $e$ be the identity of $S$. Since $S$ is a Lie ideal of $R$, for any $x \in R$, ex $-x e \in S$, and so, $e(e x-x e)=e x-x e=(e x-x e) e$. These equalities imply that $e x=e x e=x e$, or $e \in Z$. Hence $e R \oplus$ $(1-e) R=R$. Suppose $a=(1-e) a$. Then $a^{*}=(1-e) a^{*}$, so $a, a^{*} \in$ $(1-e) R$. But $e S=S \subset e R$, so $a a^{*}=a+a^{*}=0$, and $\left(a+a^{*}\right) a=$ $a^{2}=0$. Further, if $2 a=0$ then $a+a^{*}=0$ implies $a=a^{*} \in S \subset e R$. Thus $(1-e) R$ is a 2-torsion free nil ideal of index 2 .

As a result of Lemma 3.9 there is no great loss in assuming that $S$ consists of units of $R$ and that the identity of $S$ is the identity of $R$. Henceforth we shall make this assumption.

We claim that we also may as well assume that $R$ is semi-prime. If $2 R=0$, then by Lemma $3.6 R$ is semi-prime. Assume that char $S \neq$ 2 but $2 r=0$ for some $r \in R$. Then $2\left(r+r^{*}\right)=0$, so $r+r^{*}=0$. Since $2 r=0$ we have $r=r^{*} \in S$, so $r=0$. Therefore $R$ is 2-torsion free, and so, $R / N$ is semi-prime and 2-torsion free, where $N$ is the ideal of Lemma 3.6. Consider $x+N \in S(R / N)$. Thus $x+N=x^{*}+N$, and so, $x-x^{*} \in N$. Hence $2 x-\left(x+x^{*}\right)=x-x^{*} \in N$. We may conclude that $2 x+N$ is the image of $x+x^{*} \in S$. Since $2 \in S$, it is a unit in $R$, and we have $S(R / N) \cong S$. Thus in all cases we can reduce to $R$ semi-prime. With this assumption, we come to our last result.

Theorem 3.10. If $S$ is a field, * is not the identity map, and $R$ is semi-prime, then one of the following must hold:

(1) $R$ is a field and $[R: S]=2$

(2) $R=S \oplus S$ and * interchanges co-ordinates

(3) $R$ is a division ring with $[R: Z]=4$

(4) $R=S_{2}$ and char $S \neq 2$.

Proof. If $R$ is not simple let $m$ be a maximal ideal in $R$. Because nonzero elements of $S$ are units in $R, m \cap S=0$. As we have seen before, $x \in m \cap m^{*}$ implies $x x^{*}, x+x^{*} \in m \cap S=0$, so $x^{2}=0$. Further, $m \cap m^{*}$ must be 2-torsion free, so $\left(m \cap m^{*}\right)^{3}=0$. But $R$ is semi-prime, so $m \cap m^{*}=0$. Since $m$ is maximal $m+m^{*}=R$. Thus $R=R / m \oplus R / m^{*}$. If $x \in m^{*}$ then $x+m=\left(x+x^{*}\right)+m$, so $R / m=$ 
$(S+m) / m \cong S$. Hence $R / m$ is a field isomorphic to $S$. Since $R / m^{*}$ is anti-isomorphic to $R / m$, as in Theorem 3.7, we have possibility (2) of the theorem.

Assume now that $R$ is simple. Suppose first that $S \not \supset Z$ and let $c \in Z$ with $c-c^{*} \neq 0$. For any $r \in R,\left(c-c^{*}\right) r=c\left(r+r^{*}\right)-\left(c^{*} r+r^{*} c\right) \epsilon$ $c S+S$. Since $c-c^{*} \in Z$ we have that $R$ is commutative, so a field. As $*$ is not the identity map, $S$ is its fixed field and $[R: S]=2$, giving possibility (1) of the theorem.

Since $S$ is a Lie ideal of $R$, by Theorem 1.5 of [3], either $S \subset Z$ or char $R=2$ and $\operatorname{dim}_{Z} R \leqq 4$. Even if $S \subset Z$, since $x^{2}-\left(x+x^{*}\right) x+x^{*} x=0$ for any $x \in R, R$ is quadratic over $Z$, so $\operatorname{dim}_{z} R \leqq 4$ by Kaplansky's Theorem [2, p. 157]. Hence we may assume that $R$ is simple of dimension 4 over its center, for otherwise $R$ is commutative and we have case (1) as before. If char $R \neq 2$, then $S=Z$, so (3) or (4) holds. Lastly, if char $R=2$, then if $x \in R$ and $x^{2}=0$ we have $x x^{*}=x^{*} x=0$, since they are not units, and so $\left(x+x^{*}\right)^{2}=0$. Thus $x+x^{*}=0$, which implies $x=x^{*} \in S$. Therefore, we may conclude that $R$ has no nilpotent elements, and so, (4) can hold only if char $R \neq 2$.

Examples similar to those after Theorem 3.7 show that in case char $S \neq 2, R$ may have nilpotent ideals which are not direct summands even though $S$ is a field. Specifically, take $F[x] /\left(x^{2}\right)$ with $x^{*}=-x$. For a noncommutative example start with $T=x F[x, y]+y F[x, y]$. Let $I$ be the ideal generated by $x^{2}-x, y^{2}-y$, and $x y$. Set $W=T / I$ with * interchanging $x$ and $y$. Let $R=W\left\{z_{i}\right\}$ the free algebra with constants over $W$ in more than two indeterminates $\left\{z_{i}\right\}$. Let $J$ be the ideal of $R$ generated by all $x z_{i}, y z_{i}$ and all squares in the ring generated by $\left\{z_{i}\right\}$. Extend $*$ to $R / J$ by setting $z_{i}^{*}=-z_{i}$. Then the ideal $N$ of Lemma 3.6 is the ideal generated by $\left\{z_{i}\right\}$ and $S=F \cdot(x+y) \cong$ $F$.

\section{REFERENCES}

1. S. A. Amitsur, Identities in rings with involution, Israel J. of Math., (1969) 7, 63-68.

2. I. N. Herstein, Non Commutative Rings, The Carus Mathematical Monographs, Math. Assn. of Amer., 1968.

3. - Topics in Ring Theory, University of Chicago Press, Chicago, 1969.

4. N. Jacobson, Structure of Rings, Amer. Math. Soc. Colloquium Publication v. 37. Amer. Math. Society, Providence, 1964.

5, M. S. Montgomery, Lie structure of simple rings of characteristic 2, J. Algebra 15, (1970), 387-407.

Received October 11, 1971 and in revised form February 28, 1972. This work was supported by NSF Grant GP-29119X. 


\section{PACIFIC JOURNAL OF MATHEMATICS}

\section{EDITORS}

\section{H. SAMELSON}

Stanford University

Stanford, California 94305

C. R. Новву

University of Washington Seattle, Washington 98105

\section{J. DuGundJI}

Department of Mathematics University of Southern California Los Angeles, California 90007

RICHARD ARENS

University of California Los Angeles, California 90024

\section{ASSOCIATE EDITORS}
E. F. BECKENBACH
B. H. NeumanN
F. WOLF
K. YoSHIDA

\section{SUPPORTING INSTITUTIONS}

\author{
UNIVERSITY OF BRITISH COLUMBIA \\ CALIFORNIA INSTITUTE OF TECHNOLOGY \\ UNIVERSITY OF CALIFORNIA \\ MONTANA STATE UNIVERSITY \\ UNIVERSITY OF NEVADA \\ NEW MEXICO STATE UNIVERSITY \\ OREGON STATE UNIVERSITY \\ UNIVERSITY OF OREGON \\ OSAKA UNIVERSITY
}

\author{
UNIVERSITY OF SOUTHERN CALIFORNIA \\ STANFORD UNIVERSITY \\ UNIVERSITY OF TOKYO \\ UNIVERSITY OF UTAH \\ WASHINGTON STATE UNIVERSITY \\ UNIVERSITY OF WASHINGTON \\ $*{ }^{*}$
AMERICAN MATHEMATICAL SOCIETY
NAVAL WEAPONS CENTER
}

The Supporting Institutions listed above contribute to the cost of publication of this Journal, but they are not owners or publishers and have no responsibility for its content or policies.

Mathematical papers intended for publication in the Pacific Journal of Mathematics should be in typed form or offset-reproduced, (not dittoed), double spaced with large margins. Underline Greek letters in red, German in green, and script in blue. The first paragraph or two must be capable of being used separately as a synopsis of the entire paper. The editorial "we" must not be used in the synopsis, and items of the bibliography should not be cited there unless absolutely necessary, in which case they must be identified by author and Journal, rather than by item number. Manuscripts, in duplicate if possible, may be sent to any one of the four editors. Please classify according to the scheme of Math. Rev. Index to Vol, 39. All other communications to the editors should be addressed to the managing editor, Richard Arens, University of California, Los Angeles, California, 90024.

50 reprints are provided free for each article; additional copies may be obtained at cost in multiples of 50 .

The Pacific Journal of Mathematics is issued monthly as of January 1966. Regular subscription rate: $\$ 48.00$ a year (6 Vols., 12 issues). Special rate: $\$ 24.00$ a year to individual members of supporting institutions.

Subscriptions, orders for back numbers, and changes of address should be sent to Pacific Journal of Mathematics, 103 Highland Boulevard, Berkeley, California, 94708.

PUBLISHED BY PACIFIC JOURNAL OF MATHEMATICS, A NON-PROFIT CORPORATION

Printed at Kokusai Bunken Insatsusha (International Academic Printing Co., Ltd.), 270, 3-chome Totsuka-cho, Shinjuku-ku, Tokyo 160, Japan. 


\section{Pacific Journal of Mathematics}

\section{Vol. 44, No. $2 \quad$ June, 1973}

Tsuyoshi Andô, Closed range theorems for convex sets and linear liftings . . . . . . 393

Richard David Bourgin, Conically bounded sets in Banach spaces . . . . . . . . . 411

Robert Jay Buck, Hausdorff dimensions for compact sets in $R^{n} \ldots \ldots \ldots \ldots \ldots \ldots . \ldots 421$

Henry Cheng, A constructive Riemann mapping theorem ................ 435

David Fleming Dawson, Summability of subsequences and stretchings of

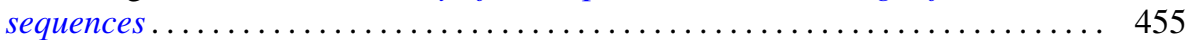

William Thomas Eaton, A two sided approximation theorem for 2-spheres ....... 461

Jay Paul Fillmore and John Herman Scheuneman, Fundamental groups of compact complete locally affine complex surfaces ....................... 487

Avner Friedman, Bounded entire solutions of elliptic equations . . . . . . . . . . . 497

Ronald Francis Gariepy, Multiplicity and the area of an $(n-1)$ continuous

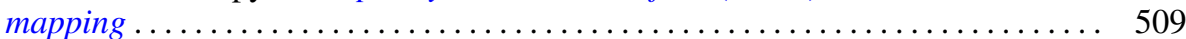

Andrew M. W. Glass, Archimedean extensions of directed interpolation groups . . . . 515

Morisuke Hasumi, Extreme points and unicity of extremum problems in $H^{1}$ on

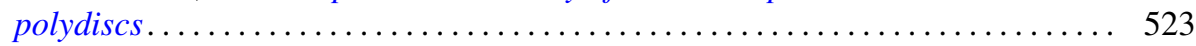

Trevor Ongley Hawkes, On the Fitting length of a soluble linear group . . . . . . 537

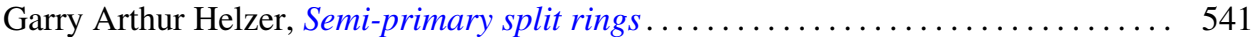

Melvin Hochster, Expanded radical ideals and semiregular ideals . . . . . . . . . 553

Keizō Kikuchi, Starlike and convex mappings in several complex variables . . . . . . 569

Charles Philip Lanski, On the relationship of a ring and the subring generated by its

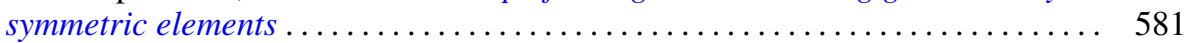

Jimmie Don Lawson, Intrinsic topologies in topological lattices and semilattices ........................................... 593

Roy Bruce Levow, Counterexamples to conjectures of Ryser and de Oliveira ...... 603

Arthur Larry Lieberman, Some representations of the automorphism group of an infinite continuous homogeneous measure algebra ..........

William George McArthur, $G_{\delta}$-diagonals and metrization theorems $\ldots .$.

James Murdoch McPherson, Wild arcs in three-space. II. An invariant of

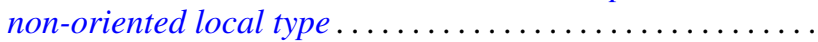

H. Millington and Maurice Sion, Inverse systems of group-valued measures ...

C. Edward Moore, Concrete semispaces and lexicographic separation of convex

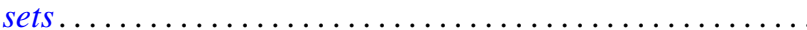

Jingyal Pak, Actions of torus $T^{n}$ on $(n+1)$-manifolds $M^{n+1}$.

Merrell Lee Patrick, Extensions of inequalities of the Laguerre and Turán type . . . . 675

Harold L. Peterson, Jr., Discontinuous characters and subgroups of finite index. . . . 683

S. P. Philipp, Abel summability of conjugate integrals . . . . . . . . . . . . . 693

R. B. Quintana and Charles R. B. Wright, On groups of exponent four satisfying an

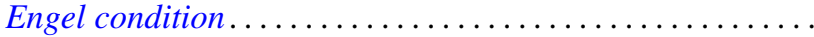

Marlon C. Rayburn, On Hausdorff compactifications. . . . . . . . . .

Martin G. Ribe, Necessary convexity conditions for the Hahn-Banach theorem in

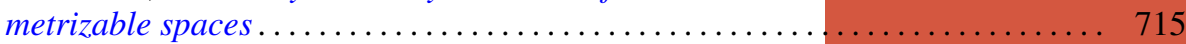

Ryōtarō Satō, On decomposition of transformations in infinite measure spaces .... 733

Peter Drummond Taylor, Subgradients of a convex function obtained from a

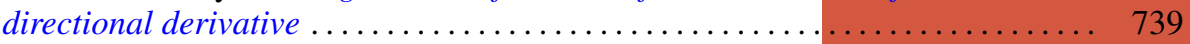

James William Thomas, A bifurcation theorem for $k$-set contractions . . . . . . . . 749 Clifford Edward Weil, A topological lemma and applications to real functions . . . . 757

Stephen Andrew Williams, A nonlinear elliptic boundary value problem . . ....... 767

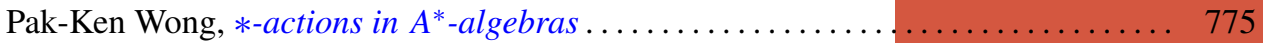

